\title{
Performance of Diversity System Output Signal in Mobile Cellular System in the Presence of $\alpha-\mu$ Short Term Fading and Gamma Long Term Fading
}

\author{
Dragana KRSTIC ${ }^{1}$, Branimir JAKSIC ${ }^{2}$, Milan GLIGORIJEVIC ${ }^{3}$, \\ Dusan STEFANOVIC ${ }^{4}$, Mihajlo STEFANOVIC ${ }^{1}$ \\ ${ }^{1}$ Faculty of Electronic Engineering, University of Nis, Aleksandra Medvedeva 14, Nis, Serbia \\ ${ }^{2}$ University of Pristina, Faculty of Technical Sciences, Kneza Milosa 7, 40000 Kosovska Mitrovica, Serbia \\ ${ }^{3}$ Criminal Police Academy, 11000 Belgrade, Serbia \\ ${ }^{4}$ College of Applied Technical Sciences, Aleksandra Medvedeva 20, 18000 Nis, Serbia \\ dragana.krstic@elfak.ni.ac.rs, branimir.jaksic@pr.ac.rs,milan.gligorijevic@mup.gov.rs, \\ dusan.stefanovic@vtsnis.edu.rs, misa.profesor@gmail.com
}

Manuscript received April 12, 2016

\begin{abstract}
In this paper, wireless mobile communication system with macrodiversity reception is considered. Macrodiversity system is consisting of macrodiversity selection combining (SC) receiver and three microdiversity $S C$ receivers. Propagation channel suffers $\alpha-\mu$ short term fading and Gamma long term fading resulting in system performance degradation. Analytical closed form expression for average level crossing rate (LCR) of macrodiversity SC receiver output signal envelope is obtained. Mathematical results are analyzed, presenting the influence of long term fading parameters and short term fading parameters on average level crossing rate. Obtained results can be used in the process of simulation and design of real-world environments mobile cellular telecommunication systems.
\end{abstract}

\section{Keywords}

$\alpha-\mu$ short term fading, Gamma long term fading, correlation, level crossing rate, macrodiversity, microdiversity, SC combining

\section{Introduction}

The received signal in wireless communication system experiences long term fading and short term fading resulting in system performance and channel capacity degradation [1]. Macrodiversity system can be used to reduce, simultaneously, long term fading effects and short term fading effects on system performance.

Several distributions can be used to describe signal envelope variation in fading channels [1], [2]. The $\alpha-\mu$ distribution and Weibull distribution present signal envelope in nonlinear and non-line-of-sight conditions in the presence of multipath fading. Weibull distribution refers to signal envelope in fading channel with one cluster and $\alpha-\mu$ distribution to small scale signal envelope variation in nonlinear multipath fading channels with two or more clusters. The $\alpha-\mu$ distribution has two parameters where parameter $\alpha$ is related to nonlinearity of propagation channel and parameter $\mu$ is related to the number of clusters in propagation channel. Rayleigh, Nakagami-m and Weibull distribution can be derived from $\alpha-\mu$ distribution. By setting $\alpha=2$, the $\alpha-\mu$ distribution reduces to Nakagami-m and Weibull distribution can be obtained from the $\alpha-\mu$ distribution by setting $\mu=1$. If $\alpha=2$ and $\mu=1$, the $\alpha-\mu$ distribution approximates Rayleigh distribution [3].

Two distributions can depict long term fading envelope power variation in shadowed fading channels: lognormal distribution and Gamma distribution. A closed solution presented in [4] shows the composite probability distribution of power levels derived from short term Rayleigh fading with superimposed long term lognormal variations of mean value, but it is not in closed form expression. The expressions for performance of wireless communication system subjected to long term fading have a closed form, when Gamma distribution describes signal envelope power variation.

The macrodiversity system has macrodiversity receiver and two or more microdiversity receivers. Macrodiversity receiver reduces long term fading and microdiversity receivers mitigate short term fading effects on system performances. In cellular mobile radio system, microdiversity receivers combine signals from multiple antennas at a base station and macrodiversity receiver deals with signals from two or more base stations distributed in a cell [5].

The second order performance measures of wireless communication system are average level crossing rate (LCR) and average fade duration (AFD). Average level crossing rate and average fade duration are important performance measures enabling dynamic analysis of wireless communication system. Average LCR is equal to average 
value of the first derivation of random process and AFD can be calculated as a ratio of outage probability and average level crossing rate [3].

In open technical literature, many papers exist which treat first and second order statistics of wireless communication system. Outage probability in shadowed fading channels is evaluated in [6] when macrodiversity and microdiversity techniques are implemented. Selection combining system over correlated Generalized-K (KG) fading channels in the presence of co-channel interference is analyzed in [7].

The infinite-series expressions for the second-order statistical measures of macro-diversity structure operating over Gamma shadowed $\kappa-\mu$ fading channels is provided in [8]. The authors focused on maximal ratio combining (MRC) at each base station (micro-diversity), and SC combining, based on output signal power values, between base stations (macro-diversity).

In [9], average LCR and AFD of macrodiversity system with macrodiversity $\mathrm{SC}$ receiver and two microdiversity SC receivers operating over Gamma shadowed Nakagami-m multipath fading environment are processed. In [10], second order performance measures of wireless system with macrodiversity reception in the presence of Gamma shadowing and Rician multipath fading are studied.

In this paper, the macrodiversity system with SC receiver and three microdiversity SC receivers which operate over Gamma shadowed $\alpha-\mu$ multipath fading channel is discussed. The closed form expression for average level crossing rate is derived. The second order statistics of macrodiversity system in Gamma shadowed $\alpha-\mu$ fading channel is not presented in available technical literature till now, according to the authors' knowledge.

\section{Level Crossing Rate of $\alpha-\mu$ Random Process}

The $\alpha-\mu$ random variable is given by dint of [11]:

$$
r_{1}=r^{\frac{2}{\alpha}} \Rightarrow r=r_{1}^{\frac{\alpha}{2}}
$$

where $r$ is Nakagami- $m$ random variable.

The first derivative of the $\alpha-\mu$ random variable is introduced by:

$$
\dot{r}_{1}=\frac{2}{\alpha} r_{1}^{\frac{2}{\alpha}-1} \dot{r} \Rightarrow \dot{r} \frac{\alpha}{2} r_{1}^{\frac{\alpha}{2}-1} \dot{r}_{1}
$$

where $\dot{r}$ is the first derivative of the Nakagami- $m$ random variable. The joint probability density function of $\alpha-\mu$ random variable and its first derivative is calculated by the next equation:

$$
p_{r_{1} \dot{r}_{1}}\left(r_{1} \dot{r}_{1}\right)=|J| p_{r \dot{r}}(r \dot{r})
$$

where Jacobian is given by means:

$$
J=\left|\begin{array}{ll}
\frac{\partial r}{\partial r_{1}} & \frac{\partial r}{\partial \dot{r}_{1}} \\
\frac{\partial \dot{r}}{\partial r_{1}} & \frac{\partial \dot{r}}{\partial \dot{r}_{1}}
\end{array}\right|=\left|\begin{array}{cc}
\frac{\alpha}{2} r_{1}^{\frac{\alpha}{2}-1} & 0 \\
0 & \frac{\alpha}{2} r_{1}^{\frac{\alpha}{2}-1}
\end{array}\right|=\frac{\alpha^{2}}{4} r_{1}^{\alpha-1} .
$$

The joint probability density function of Nakagami- $m$ random variable $r$ and its first derivative $\dot{r}$ is performed by:

$$
p_{r \dot{r}}(r \dot{r})=\frac{2}{\Gamma(m)}\left(\frac{m}{\Omega}\right)^{\alpha m} r^{2 m-1} \mathrm{e}^{-\frac{m}{\Omega} r^{2}} \cdot \frac{1}{\sqrt{2 \pi} \beta} \mathrm{e}^{-\frac{\dot{r}^{2}}{2 \beta^{2}}}
$$

where $\beta^{2}=\pi^{2} f_{\mathrm{m}}^{2} \Omega / m, f_{\mathrm{m}}$ is maximal Doppler frequency, $m$ is shape parameter and $\Omega$ is power of Nakagami- $m$ random variable $r$.

After substituting (4) and (5) in (3), the expression for the joint probability density function becomes:

$$
\begin{aligned}
& p_{r_{1} \dot{r}_{1}}\left(r_{1} \dot{r}_{1}\right)= \\
& \frac{\alpha^{2}}{4} r_{1}^{\alpha-2} \frac{2}{\Gamma(m)}\left(\frac{m}{\Omega}\right)^{\alpha m} r_{1}^{\alpha m-\frac{\alpha}{2}} \mathrm{e}^{-\frac{m}{\Omega} r_{1}^{\alpha}} \frac{1}{\sqrt{2 \pi} \beta} \mathrm{e}^{-\frac{\alpha^{2}}{8 \beta^{2}} r_{1}^{\alpha-2} \dot{r}_{1}^{2}}
\end{aligned}
$$

Level crossing rate of $\alpha-\mu$ random process can be calculated as the first derivative of $\alpha-\mu$ random process:

$$
N_{r_{1}}=\int_{0}^{\infty} \mathrm{d} \dot{r}_{1} \dot{r}_{1} p_{r_{1} \dot{r}_{1}}\left(r_{1} \dot{r}_{1}\right)=\frac{\sqrt{2 \pi} f_{m}}{\Gamma(m)}\left(\frac{m}{\Omega}\right)^{\alpha m-\frac{1}{2}} r_{1}^{\alpha m-\frac{\alpha}{2}} \mathrm{e}^{-\frac{m}{\Omega} r_{1}^{\alpha}} .
$$

$\Gamma(\cdot)$ denotes the Gamma function.

The SC receiver processing over independent indentical $\alpha-\mu$ multipath channel is analyzed. Signal envelopes at inputs of SC receiver are denoted with $x_{1}$ and $x_{2}$, and SC receiver output signal envelope is $x$.

The joint probability density function of SC receiver output signal and its first derivative is performed by:

$$
\begin{aligned}
p_{x \dot{x}}(x \dot{x}) & =p_{x_{1} \dot{x}_{1}}(x \dot{x}) \mathrm{F}_{x_{2}}(x)+p_{x_{2} \dot{x}_{2}}(x \dot{x}) \mathrm{F}_{x_{1}}(x)= \\
& =2 p_{x_{1} \dot{x}_{1}}(x \dot{x}) \mathrm{F}_{x_{2}}(x)
\end{aligned}
$$

where $\mathrm{F}_{x_{2}}(x)$ is cumulative distribution function of $\alpha-\mu$ random variable shown by

$$
F_{x_{2}}(x)=\frac{1}{\Gamma(m)} \gamma\left(m, \frac{m}{\Omega} x^{\alpha}\right),
$$

with $\gamma(\cdot)$ representing the lower incomplete Gamma function described by:

$$
\gamma(n, x)=\frac{1}{n} x^{n} e^{-x}{ }_{1} \mathrm{~F}_{1}(1, n+1, x)=\frac{1}{n} x^{n} e^{-x} \sum_{i=0}^{\infty} \frac{n !}{(n+i) !} x^{i}
$$

where ${ }_{1} \mathrm{~F}_{1}$ is the Kummer confluent hypergeometric function [12].

Average level crossing rate of SC receiver output signal envelope can be calculated using the formula: 


$$
\begin{aligned}
N_{x} & =\int_{0}^{\infty} \mathrm{d} \dot{x} \dot{x} p_{x \dot{x}}(x \dot{x})=\int_{0}^{\infty} \mathrm{d} \dot{x} \dot{x} 2 p_{x_{1} \dot{x}_{1}}(x \dot{x}) \mathrm{F}_{x_{2}}(x)= \\
& =2 \mathrm{~F}_{x_{2}}(x) \int_{0}^{\infty} \mathrm{d} \dot{x} \dot{x} p_{x_{1} \dot{x}_{1}}(x \dot{x})=2 \mathrm{~F}_{x_{2}}(x) N_{x_{1}}
\end{aligned}
$$

where $N_{x_{1}}$ is displayed with (7).

Cumulative distribution function of $\mathrm{SC}$ receiver output signal is described by:

$$
\mathrm{F}_{x}=\mathrm{F}_{x_{1}}(x) \mathrm{F}_{x_{2}}(x)=\left(\mathrm{F}_{x_{2}}(x)\right)^{2}
$$

where $\mathrm{F}_{x_{2}}(x)$ is cumulative distribution function of $\alpha-\mu$ random variable shown by (9).

\section{Average Level Crossing Rate of Macrodiversity SC Receiver Output Signal}

Macrodiversity system SC receiver with three microdiversity SC receivers is considered. The system that is being considered is shown in Fig. 1. Received signal is subjected to correlated Gamma long term fading and $\alpha-\mu$ short term fading. Signal envelopes at inputs of the first microdiversity SC receiver are denoted with $x_{1}$ and $x_{2}$, at inputs of the second microdiversity $\mathrm{SC}$ receiver with $y_{1}$ and $y_{2}$, and the inputs of the third microdiversity receiver with $z_{1}$ and $z_{2}$. Signal envelopes at output of microdiversity SC receivers are denoted with $x, y$ and $z$, and output of macrodiversity SC receiver with $w$. The average powers of signal envelope at inputs of microdiversity SC receivers are denoted with $\Omega_{1}, \Omega_{2}$ and $\Omega_{3}$.

Random variables $\Omega_{1}, \Omega_{2}$ and $\Omega_{3}$ follow Gamma distribution and they may be presented by:

$$
\begin{aligned}
& p_{\Omega_{1} \Omega_{2} \Omega_{3}}\left(\Omega_{1} \Omega_{2} \Omega_{3}\right)= \\
& \frac{1}{\Gamma(c)\left(1-\rho^{2}\right) \rho^{c-1} \Omega_{0}^{c+2}}\left(\Omega_{1} \Omega_{3}\right)^{\frac{c-1}{2}} \mathrm{e}^{-\frac{\Omega_{1}+\Omega_{3}+\Omega_{2}(1+\rho)}{\Omega_{0}(1-\rho)}} . \\
& \mathrm{I}_{c-1}\left(\frac{2 \sqrt{\rho}}{\Omega_{0}(1-\rho)} \sqrt{\Omega_{1} \Omega_{2}}\right) \mathrm{I}_{c-1}\left(\frac{2 \sqrt{\rho}}{\Omega_{0}(1-\rho)} \sqrt{\Omega_{2} \Omega_{3}}\right)= \\
& \frac{1}{\Gamma(c)\left(1-\rho^{2}\right) \rho^{c-1} \Omega_{0}^{c+2}} \cdot \sum_{i_{1}=0}^{\infty}\left(\frac{\sqrt{\rho}}{\Omega_{0}(1-\rho)}\right)^{2 i_{1}+c-1} . \\
& \frac{1}{i_{1} ! \Gamma\left(i_{1}+c\right)} \sum_{i_{2}=0}^{\infty}\left(\frac{\sqrt{\rho}}{\Omega_{0}(1-\rho)}\right)^{2 i_{2}+c-1} \frac{1}{i_{2} ! \Gamma\left(i_{2}+c\right)} . \\
& \Omega_{1}^{i_{1}+c-1} \Omega_{2}^{i_{1}+i_{2}+c-1} \Omega_{3}^{i_{2}+c-1} \mathrm{e}^{-\frac{\Omega_{1}+\Omega_{2}(1+\rho)+\Omega_{3}}{\Omega_{0}(1-\rho)}}
\end{aligned}
$$

where $\mathrm{I}_{n}(\cdot)$ is modified Bessel function of the first kind, order $n$ and argument $x, \Omega_{0}$ is mean square of signal power variation, $\rho$ correlation coefficient, and $c$ Gamma shadowing severity.

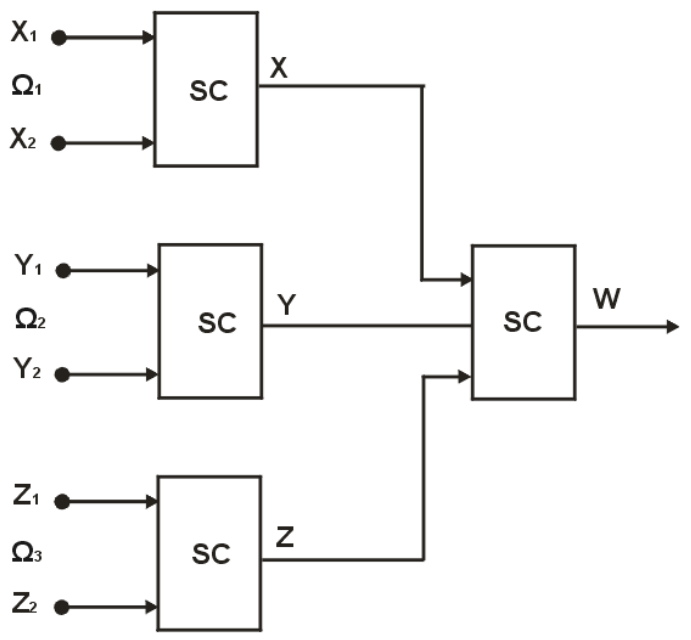

Fig. 1. Macrodiversity system with three microdiversity SC receivers.

Macrodiversity SC receiver selects microdiversity SC receiver with the highest signal envelope power at inputs to provide service to user. Therefore, average level crossing rate of macrodiversity $\mathrm{SC}$ receiver output signal envelope can be calculated using:

$$
\begin{aligned}
N_{w}= & \int_{0}^{\infty} \mathrm{d} \Omega_{1} \int_{0}^{\Omega_{1}} \mathrm{~d} \Omega_{2} \int_{0}^{\Omega_{1}} \mathrm{~d} \Omega_{3} N_{x / \Omega_{1}} p_{\Omega_{1} \Omega_{2} \Omega_{3}}\left(\Omega_{1} \Omega_{2} \Omega_{3}\right)+ \\
& \int_{0}^{\infty} \mathrm{d} \Omega_{2} \int_{0}^{\Omega_{2}} \mathrm{~d} \Omega_{1} \int_{0}^{\Omega_{2}} \mathrm{~d} \Omega_{3} N_{x / \Omega_{2}} p_{\Omega_{1} \Omega_{2} \Omega_{3}}\left(\Omega_{1} \Omega_{2} \Omega_{3}\right)+ \\
& \int_{0}^{\infty} \mathrm{d} \Omega_{3} \int_{0}^{\Omega_{3}} \mathrm{~d} \Omega_{1} \int_{0}^{\Omega_{3}} \mathrm{~d} \Omega_{2} N_{x / \Omega_{3}} p_{\Omega_{1} \Omega_{2} \Omega_{3}}\left(\Omega_{1} \Omega_{2} \Omega_{3}\right)= \\
= & I_{1}+I_{2}+I_{3}=2 I_{1}+I_{2}
\end{aligned}
$$

where $N_{x_{1} / \Omega_{1}}$ and $N_{x_{2} / \Omega_{2}}$ are given with (11) and $p_{\Omega_{1} \Omega_{2} \Omega_{3}}\left(\Omega_{1} \Omega_{2} \Omega_{3}\right)$ is given by (13).

Integral $I_{1}$ is equal to:

$$
\begin{aligned}
I_{1}= & \int_{0}^{\infty} \mathrm{d} \Omega_{1} \int_{0}^{\Omega_{1}} \mathrm{~d} \Omega_{2} \int_{0}^{\Omega_{1}} \mathrm{~d} \Omega_{3} N_{x / \Omega_{1}} p_{\Omega_{1} \Omega_{2} \Omega_{3}}\left(\Omega_{1} \Omega_{2} \Omega_{3}\right)= \\
& \int_{0}^{\infty} \mathrm{d} \Omega_{1} \int_{0}^{\Omega_{1}} \mathrm{~d} \Omega_{2} \int_{0}^{\Omega_{1}} \mathrm{~d} \Omega_{3} \frac{2 f_{m} \sqrt{2 \pi}}{\Gamma(\mu)^{2}}\left(\frac{\mu}{\Omega_{1}}\right)^{\mu-\frac{1}{2}} \cdot w^{\alpha \mu-\frac{\alpha}{2}} \mathrm{e}^{-\frac{\mu}{\Omega_{1}} w^{\alpha}} . \\
& \gamma\left(\mu, \frac{\mu}{\Omega_{1}} w^{\alpha}\right) \frac{1}{\Gamma(c)\left(1-\rho^{2}\right) \rho^{c-1} \Omega_{0}^{c+2}} . \\
& \sum_{i_{1}=0}^{\infty}\left(\frac{\sqrt{\rho}}{\Omega_{0}(1-\rho)}\right)^{2 i_{1}+c-1} \frac{1}{i_{1} ! \Gamma\left(i_{1}+c\right)} . \\
& \sum_{i_{2}=0}^{\infty}\left(\frac{\sqrt{\rho}}{\Omega_{0}(1-\rho)}\right)^{i_{2}+c-1} \frac{1}{i_{2} ! \Gamma\left(i_{2}+c\right)} . \\
& \Omega_{1}^{i_{1}+c-1} \Omega_{2}^{i_{1}+i_{2}+c-1} \Omega_{3}^{i_{2}+c-1} \mathrm{e}^{-\frac{\Omega_{1}+\Omega_{3}+\Omega_{2}(1+\rho)}{\Omega_{0}(1-\rho)}},
\end{aligned}
$$


or

$I_{1}=\frac{2 f_{m} \sqrt{2 \pi}}{\Gamma(\mu)^{2}} \mu^{\mu-\frac{1}{2}} w^{\alpha \mu-\frac{\alpha}{2}} \frac{1}{\Gamma(c)\left(1-\rho^{2}\right) \rho^{c-1} \Omega_{0}^{c+2}}$.

$\sum_{i_{1}=0}^{\infty}\left(\frac{\sqrt{\rho}}{\Omega_{0}(1-\rho)}\right)^{2 i_{1}+c-1} \frac{1}{i_{1} ! \Gamma\left(i_{1}+c\right)} \sum_{i_{2}=0}^{\infty}\left(\frac{\sqrt{\rho}}{\Omega_{0}(1-\rho)}\right)^{2 i_{2}+c-1}$.

$\frac{1}{i_{2} ! \Gamma\left(i_{2}+c\right)} \cdot \int_{0}^{\infty} \mathrm{d} \Omega_{1} \Omega_{1}^{i_{1}+c-1} \Omega_{1}^{-\mu+\frac{1}{2}} \gamma\left(\mu, \frac{\mu}{\Omega_{1}} w^{\alpha}\right) \mathrm{e}^{-\frac{\mu}{\Omega_{1}} w^{\alpha}}$.

$\mathrm{e}^{-\frac{\Omega_{1}}{\Omega_{0}(1-\rho)}} \cdot \int_{0}^{\Omega_{1}} \mathrm{~d} \Omega_{2} \Omega_{2}^{i_{1}+i_{2}+c-1} \mathrm{e}^{-\frac{\Omega_{2}(1+\rho)}{\Omega_{0}(1-\rho)}} \cdot \int_{0}^{\Omega_{1}} \mathrm{~d} \Omega_{3} \Omega_{3}^{i_{2}+c-1} \mathrm{e}^{-\frac{\Omega_{3}}{\Omega_{0}(1-\rho)}}$

After using [12] for resolving the second and third integrals in (16), $I_{1}$ becomes:

$I_{1}=\frac{2 f_{m} \sqrt{2 \pi}}{\Gamma(\mu)^{2}} \mu^{\mu-\frac{1}{2}} w^{\alpha \mu-\frac{\alpha}{2}} \frac{1}{\Gamma(c)\left(1-\rho^{2}\right) \rho^{c-1} \Omega_{0}^{c+2}}$

$\sum_{i_{1}=0}^{\infty}\left(\frac{\sqrt{\rho}}{\Omega_{0}(1-\rho)}\right)^{2 i_{1}+c-1} \frac{1}{i_{1} ! \Gamma\left(i_{1}+c\right)} \sum_{i_{2}=0}^{\infty}\left(\frac{\sqrt{\rho}}{\Omega_{0}(1-\rho)}\right)^{2 i_{2}+c-1}$

$\frac{1}{i_{2} ! \Gamma\left(i_{2}+c\right)} \cdot \int_{0}^{\infty} \mathrm{d} \Omega_{1} \Omega_{1}^{i_{1}+c-1} \Omega_{1}^{-\mu+\frac{1}{2}} \mathrm{e}^{-\frac{\mu}{\Omega_{1}} w^{\alpha}-\frac{\Omega_{1}}{\Omega_{0}(1-\rho)}}$.

$\left(\frac{\Omega_{0}(1-\rho)}{1+\rho}\right)^{i_{1}+i_{2}+c}\left(\Omega_{0}(1-\rho)\right)^{i_{2}+c} \cdot \gamma\left(\mu, \frac{\mu}{\Omega_{1}} w^{\alpha}\right)$.

$\gamma\left(i_{1}+i_{2}+c, \frac{(1+\rho)}{\Omega_{0}(1-\rho)} \Omega_{1}\right) \cdot \gamma\left(i_{2}+c, \frac{1}{\Omega_{0}(1-\rho)} \Omega_{1}\right)$

with $\gamma(\cdot)$ defined in (10). a form:

After developing Gamma function, $I_{1}$ is obtained in

$I_{1}=\frac{2 f_{m} \sqrt{2 \pi}}{\Gamma(\mu)^{2}} \mu^{\mu-\frac{1}{2}} w^{\alpha \mu-\frac{\alpha}{2}} \frac{1}{\Gamma(c)\left(1-\rho^{2}\right) \rho^{c-1} \Omega_{0}^{c+2}}$.

$\sum_{i_{1}=0}^{\infty}\left(\frac{\sqrt{\rho}}{\Omega_{0}(1-\rho)}\right)^{2 i_{1}+c-1} \frac{1}{i_{1} ! \Gamma\left(i_{1}+c\right)} \sum_{i_{2}=0}^{\infty}\left(\frac{\sqrt{\rho}}{\Omega_{0}(1-\rho)}\right)^{2 i_{2}+c-1}$.

$\frac{1}{i_{2} ! \Gamma\left(i_{2}+c\right)} \cdot \frac{1}{\mu} \mu^{\mu} w^{\mu \alpha} \sum_{j_{1}=0}^{\infty} \frac{\mu !}{\left(\mu+j_{1}\right) !} \mu^{j_{1}} w^{\alpha j_{1}} \frac{1}{i_{1}+i_{2}+c}$.

$\sum_{j_{2}=0}^{\infty} \frac{\left(i_{1}+i_{2}+c\right) !}{\left(i_{1}+i_{2}+c+j_{2}\right) !}\left(\frac{(1+\rho)}{\Omega_{0}(1-\rho)}\right)^{j_{2}}$.

$\frac{1}{i_{2}+c} \sum_{j_{3}=0}^{\infty} \frac{\left(i_{2}+c\right) !}{\left(i_{2}+c+j_{3}\right) !} \frac{1}{\left(\Omega_{0}(1-\rho)\right)^{j_{3}}}$.

$\int_{0}^{\infty} \mathrm{d} \Omega_{1} \Omega_{1}^{2 i_{1}+3 c-2 \mu+2 i_{2}-j_{1}+j_{2}+j_{3}-\frac{1}{2}} \mathrm{e}^{-\frac{2 \mu w^{\alpha}}{\Omega_{1}}-\frac{(2+\rho)}{\Omega_{0}(1-\rho)} \Omega_{1}}$.

By using [12], it is obtained:
$I_{1}=\frac{2 f_{m} \sqrt{2 \pi}}{\Gamma(\mu)^{2}} \mu^{\mu-\frac{1}{2}} w^{\alpha \mu-\frac{\alpha}{2}} \frac{1}{\Gamma(c)\left(1-\rho^{2}\right) \rho^{c-1} \Omega_{0}^{c+2}}$.

$\sum_{i_{1}=0}^{\infty}\left(\frac{\sqrt{\rho}}{\Omega_{0}(1-\rho)}\right)^{2 i_{1}+c-1} \frac{1}{i_{1} ! \Gamma\left(i_{1}+c\right)} \sum_{i_{2}=0}^{\infty}\left(\frac{\sqrt{\rho}}{\Omega_{0}(1-\rho)}\right)^{2 i_{2}+c-1}$.

$\frac{1}{i_{2} ! \Gamma\left(i_{2}+c\right)} \cdot \mu^{\mu-1} w^{\mu \alpha} \sum_{j_{1}=0}^{\infty} \frac{\mu !}{\left(\mu+j_{1}\right) !} \mu^{j_{1}} w^{\alpha j_{1}}$.

$\frac{1}{i_{1}+i_{2}+c} \sum_{j_{2}=0}^{\infty} \frac{\left(i_{1}+i_{2}+c\right) !}{\left(i_{1}+i_{2}+c+j_{2}\right) !}\left(\frac{(1+\rho)}{\Omega_{0}(1-\rho)}\right)^{j_{2}} \frac{1}{i_{2}+c}$.

$\sum_{j_{3}=0}^{\infty} \frac{\left(i_{2}+c\right) !}{\left(i_{2}+c+j_{3}\right) !} \frac{1}{\left(\Omega_{0}(1-\rho)\right)^{j_{3}}}$.

$\left(\frac{2 \mu w^{\alpha} \Omega_{0}(1-\rho)}{(2+\rho)}\right)^{i_{1}+\frac{3}{2} c-\mu+i_{2}-\frac{j_{1}}{2}+\frac{j_{2}}{2}+\frac{j_{3}}{2}+\frac{1}{2}}$.

$\mathrm{K}_{2 i_{1}+3 c-2 \mu+2 i_{2}-j_{1}+j_{2}+j_{3}+1}\left(2 \sqrt{\frac{2 \mu w^{\alpha}(2+\rho)}{\Omega_{0}(1-\rho)}}\right)$

where $K_{n}(x)$ is modified Bessel function of the second kind, order $n$ and argument $x$ [12].

Integral $I_{2}$ is equal to:

$I_{2}=\int_{0}^{\infty} \mathrm{d} \Omega_{2} \int_{0}^{\Omega_{2}} \mathrm{~d} \Omega_{1} \int_{0}^{\Omega_{2}} \mathrm{~d} \Omega_{3} N_{x / \Omega_{2}} p_{\Omega_{1} \Omega_{2} \Omega_{3}}\left(\Omega_{1} \Omega_{2} \Omega_{3}\right)=$

$\int_{0}^{\infty} \mathrm{d} \Omega_{2} \int_{0}^{\Omega_{2}} \mathrm{~d} \Omega_{1} \int_{0}^{\Omega_{2}} \mathrm{~d} \Omega_{3} \frac{2 f_{m} \sqrt{2 \pi}}{\Gamma(\mu)^{2}}\left(\frac{\mu}{\Omega_{2}}\right)^{\mu-\frac{1}{2}} \cdot w^{\alpha \mu-\frac{\alpha}{2}} \mathrm{e}^{-\frac{\mu w^{\alpha}}{\Omega_{2}}}$.

$\gamma\left(\mu, \frac{\mu w^{\alpha}}{\Omega_{2}}\right) \frac{1}{\Gamma(c)\left(1-\rho^{2}\right) \rho^{c-1} \Omega_{0}^{c+2}} \cdot \sum_{i_{2}=0}^{\infty}\left(\frac{\sqrt{\rho}}{\Omega_{0}(1-\rho)}\right)^{2 i_{2}+c-1}$

$\frac{1}{i_{2} ! \Gamma\left(i_{2}+c\right)} \Omega_{1}^{i_{1}+c-1} \Omega_{2}^{i_{1}+i_{2}+c-1} \Omega_{3}^{i_{2}+c-1} \mathrm{e}^{-\frac{\Omega_{1}+\Omega_{2}(1+\rho)+\Omega_{3}}{\Omega_{0}(1-\rho)}}$.

After using the procedure for solving $I_{1}$ and $I_{2}$, the integral $I_{2}$ is obtained in a form given in the next equation:

$I_{2}=\frac{2 f_{m} \sqrt{2 \pi}}{\Gamma(\mu)^{2}} \mu^{\mu-\frac{1}{2}} w^{\alpha \mu-\frac{\alpha}{2}} \frac{1}{\Gamma(c)\left(1-\rho^{2}\right) \rho^{c-1} \Omega_{0}^{c+2}}$

$\sum_{i_{1}=0}^{\infty}\left(\frac{\sqrt{\rho}}{\Omega_{0}(1-\rho)}\right)^{2 i_{1}+c-1} \frac{1}{i_{1} ! \Gamma\left(i_{1}+c\right)} \sum_{i_{2}=0}^{\infty}\left(\frac{\sqrt{\rho}}{\Omega_{0}(1-\rho)}\right)^{2 i_{2}+c-1}$.

$\frac{1}{i_{2} ! \Gamma\left(i_{2}+c\right)} \cdot \frac{\mu^{\mu} w^{\mu \alpha}}{\mu} \sum_{j_{1}=0}^{\infty} \frac{\mu !}{\left(\mu+j_{1}\right) !} \mu^{j_{1}} w^{\alpha j_{1}} \frac{1}{i_{1}+c}$.

$\sum_{j_{2}=0}^{\infty} \frac{\left(i_{1}+c\right) !}{\left(i_{1}+c+j_{2}\right) !} \frac{1}{\left(\Omega_{0}(1-\rho)\right)^{j_{2}}}$.

$\cdot \frac{1}{i_{2}+c} \sum_{j_{3}=0}^{\infty} \frac{\left(i_{2}+c\right) !}{\left(i_{2}+c+j_{3}\right) !} \frac{1}{\left(\Omega_{0}(1-\rho)\right)^{j_{3}}}$. 


$$
\begin{aligned}
& \left(\frac{2 \mu w^{\alpha} \Omega_{0}(1-\rho)}{3+\rho}\right)^{i_{1}+i_{2}+\frac{3}{2} c-\mu-\frac{j_{1}}{2}+\frac{j_{2}}{2}+\frac{j_{3}}{2}+\frac{1}{4}} \\
& K_{2 i_{1}+2 i_{2}+3 c-2 \mu-j_{1}+j_{2}+j_{3}+\frac{1}{2}}\left(2 \sqrt{\frac{2 \mu w^{\alpha}(3+\rho)}{\Omega_{0}(1-\rho)}}\right) .
\end{aligned}
$$

\section{Numerical Results}

In Fig. 2, the normalized level crossing rate of macrodiversity SC receiver output signal envelope is plotted versus macrodiversity $\mathrm{SC}$ receiver output signal envelope $w$, for different values of number of clusters $\mu$ and nonlinearity parameter $\alpha$.

One can see from this figure that an increase of output signal envelope $w$ first leads to the increasing of the average level crossing rate. After reaching the maximum, this leads to rapid decline of average level crossing rate. With bigger values of nonlinearity coefficient $\alpha$ and number of clusters $\mu$, LCR is declining.

In Fig. 3, the normalized level crossing rate of macrodiversity SC receiver output signal envelope is plotted versus correlation coefficient $\rho$, for different values of Gamma fading severity $c$. The other parameters values are: nonlinearity parameter $\alpha=0.5$, number of clusters $\mu=2$, power $\Omega_{1}=1$ and normalized output signal envelope $w=1$. When the correlation coefficient increases, then the average level crossing rate increases. On the contrary, with increasing of Gamma shadowing severity $c$, the average level crossing rate is getting smaller.

It is necessary to choose such system parameters to achieve smaller values of average level crossing rate, because the system performances are better in that case.

\section{Conclusion}

Macrodiversity system including SC macrodiversity receiver and three microdiversity SC receivers working over shadowed multipath fading channel is analyzed. The received signal experiences correlated Gamma large scale fading and $\alpha-\mu$ small scale fading. Macrodiversity SC receiver reduces Gamma long term fading effects and microdiversity SC receivers mitigate $\alpha-\mu$ short term fading effects on level crossing rate. The $\alpha-\mu$ fading channel is general fading channel. When $\mu$ goes to 1 , Gamma shadowed $\alpha-\mu$ multipath channel becomes Gamma shadowed Weibull channel. When parameter $\alpha$ is 2 and parameter $\mu$ is 1 , Gamma shadowed $\alpha-\mu$ multipath channel becomes Gamma shadowed Rayleigh multipath channel. In this paper, average level crossing rate of macrodiversity $\mathrm{SC}$ receiver output signal envelope is calculated. Obtained expression converges for any values of Gamma severity parameter. For $\mu=1$, the obtained expression becomes the expression for average level crossing rate of macrodiversity system in the presence of Weibull fading.

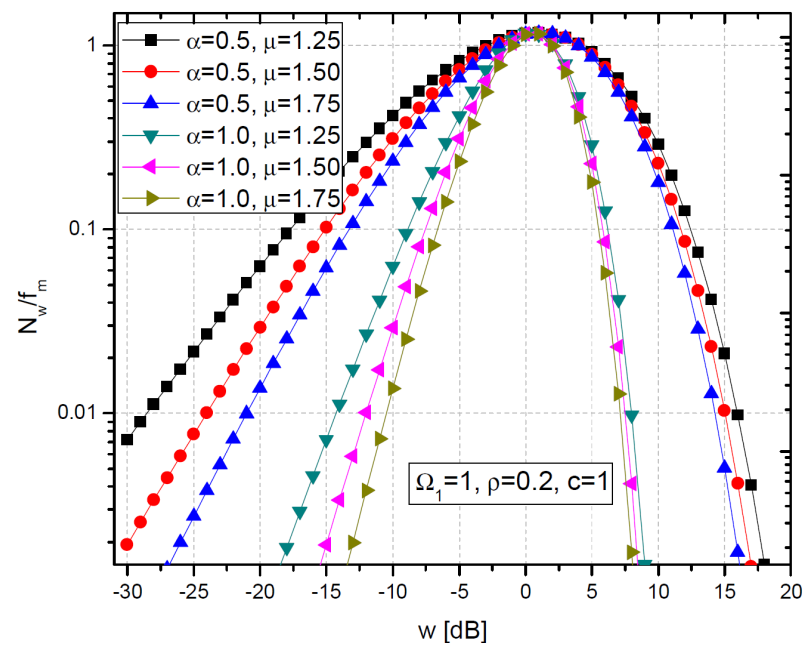

Fig. 2. Level crossing rate of macrodiversity SC receiver output signal envelope for different values of number of clusters $\mu$ and nonlinearity parameter $\alpha$.

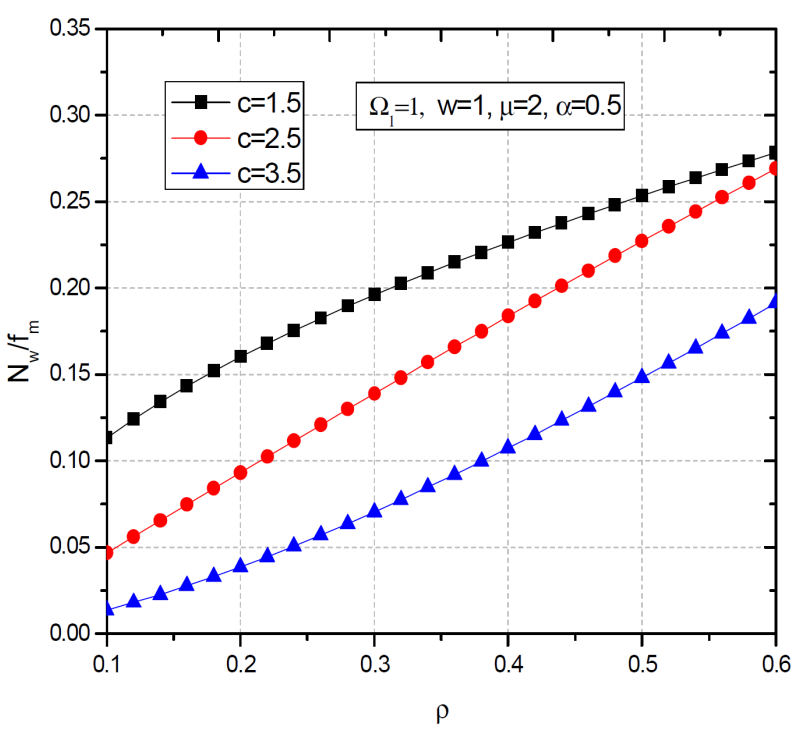

Fig. 3. Level crossing rate of macrodiversity SC receiver output signal envelope versus correlation coefficient $\rho$.

Numerical results are presented graphically to illustrate the influence of shadowing severity, multipath severity and correlation of shadowing on average level crossing rate. The system performance is better for lower values of average level crossing rate of output signal envelope. Average level crossing rate increases as Gamma long term fading severity and $\alpha-\mu$ short term fading severity decreases. When correlation coefficient goes to 1 , macrodiversity system becomes microdiversity system. In this case, the same signal occurs at all three base stations.

\section{Acknowledgments}

This paper has been supported and partially funded by the Ministry of Education, Science and Technological Development of the Republic of Serbia under projects TR33035 and III-44006. 


\section{References}

[1] GOLDSMITH, A. Wireless Communications. Cambridge (UK): Cambridge University Press, 2005. ISBN-13: 978-0521837163

[2] SIMON, M. K., ALOUNI, M. S. Digital Communication over Fading Channels. $2^{\text {nd }}$ ed. New Jersey: Wiley, 2005. ISBN: 978-0471-64953-3

[3] PANIC, S., STEFANOVIC, M., ANASTASOV, J., SPALEVIC. P. Fading and Interference Mitigation in Wireless Communications. USA: CRC Press, 2013. ISBN: 9781466508415

[4] HANSEN, F., MENO, F. I. Mobile fading-Rayleigh and lognormal superimposed. IEEE Transactions on Vehicular Technology, 1977, vol. 26 , no. 4, p. 332-335. DOI: 10.1109/T-VT.1977.23703

[5] MUKHERJEE, S., AVIDOR, D. Effect of microdiversity and correlated macrodiversity on outages in a cellular system. IEEE Transactions on Wireless Technology, 2003, vol. 2, no. 1, p. 50 to 59. DOI: $10.1109 /$ TWC.2002.806363

[6] SHANKAR, P. M. Macrodiversity and microdiversity in correlated shadowed fading channels. IEEE Transactions on Vehicular Technology, 2009, vol. 58, no. 2, p. 727-732. DOI: 10.1109/TVT.2008.926622

[7] NIKOLIC, B., STEFANOVIC, M., PANIĆ, S., ANASTASOV, J., MILOSEVIC, B. Closed-form expressions for selection combining system statistics over correlated Generalized-K (KG) fading channels in the presence of interference. ETRI Journal, 2011, vol. 33, no. 3, 2011, p. 320-325. DOI: 10.4218/etrij.11.0110.0311

[8] PANIC, S., STEFANOVIC, D., PETROVIC, I., STEFANOVIC, M., ANASTASOV, J., KRSTIC, D. Second-order statistics of selection macro-diversity system operating over Gamma shadowed $\kappa-\mu$ fading channels. EURASIP Journal on Wireless Communications and Networking, 2011, Article ID 151, p. 1-7. DOI: 10.1186/1687-1499-2011-151

[9] STEFANOVIC, D., PANIC, S., SPALEVIC, P. Second-order statistics of SC macrodiversity system operating over Gamma shadowed Nakagami-m fading channels. AEU International Journal of Electronics and Communications, 2011, vol. 65, no. 5 , p. 413-418. DOI: 10.1016/j.aeue.2010.05.001

[10] SEKULOVIC, N., STEFANOVIĆ, M. Performance analysis of system with micro- and macrodiversity reception in correlated Gamma shadowed Rician fading channels. Wireless Personal Communications, 2012, vol. 65, no. 1, p. 143-156. DOI: $10.1007 / \mathrm{s} 11277-011-0232-8$

[11] YACOUB, M. D. The $\alpha-\mu$ distribution: A physical fading model for the stacy distribution. IEEE Transactions on Vehicular Technology, 2007, vol. 56, no. 1, p. 27-34. DOI: 10.1109/TVT.2006.883753

[12] GRADSHTEYN, I. S., RYZHIK, I. M. Table of Integrals, Series and Products. San Diego: Academic Press, 2000. ISBN: 0-12294757-6

\section{About the Authors ...}

Dragana KRSTIC (corresponding author) was born in Pirot, Serbia, in 1966. She received the B.Sc, M.Sc. and $\mathrm{PhD}$. degrees in Electrical Engineering from the Faculty of Electronic Engineering, University of Nis, Serbia in 1990, 1998, and 2006, respectively. She is with the Faculty of
Electronic Engineering, University of Nis, since 1990. Her field of interest includes telecommunications theory, optical communication systems, wireless, mobile and satellite communication systems. As author/co-author, she wrote about 200 scientific research papers, of which about 40 are printed in international journals, several in national journals, and more than 100 are referred at international symposia and conferences. Dr Krstic had more plenary and keynote lectures, panels and tutorials by invitation at international conferences. She is also the member of editorial boards of a few international journals and reviewer for many. Also, she is a member of the technical program committees or reviewer for more than 50 conferences.

Branimir JAKSIC was born in Kosovska Mitrovica, Serbia, in 1984. He received the B.Sc. and M.Sc. degrees from the Faculty of Technical Sciences, University of Pristina in 2008, and 2011, respectively, and Ph.D. degree in Electrical Engineering from the Faculty of Electronic Engineering, University of Nis, Serbia, in 2015. Now, he is working at the University of Pristina, Faculty of Technical Sciences, Kosovska Mitrovica. His area of research includes statistical communications theory and optical telecommunications.

Milan GLIGORIJEVIC was born in Jagodina, Serbia, in 1965. He finished the Military Academy in Belgrade, Dept. of Telecommunications, in 1988. He received M.Sc. and Ph.D. from the Faculty of Security in Belgrade in 2008 and 2012, respectively. From 2012, he is partly employed as an assistant professor at the Criminal Police Academy, Belgrade. His research interests include criminal-police and security skills, as well as communications and crypto protection.

Dusan STEFANOVIC was born in Nis, Serbia, in 1979. He received the B.Sc. and Ph.D. degrees in Electrical Engineering from the Faculty of Electronic Engineering, Dept. of Telecommunications, University of Nis, Serbia. $\mathrm{He}$ is working as Professor at the Professional Studies at College of Applied Technical Sciences in Nis. His research interests include wireless and mobile telecommunication systems.

Mihajlo STEFANOVIC was born in Nis, Serbia, in 1947. He received the B.Sc., M.Sc. and Ph.D. degrees in Electrical Engineering from the Faculty of Electronic Engineering, Dept. of Telecommunications, University of Nis, Serbia, in 1971, 1976 and 1979, respectively. His primary research interests are statistical communication theory, optical and wireless communications. He has written or coauthored a great number of journal publications. He has written five monographs, too. He was a mentor to hundreds of graduates, for dozens of master's theses and doctoral dissertations, and many times a member of the committee for the defense of these works. Now, dr Stefanović is a retired professor. 